\title{
ESTUDO DO IMPACTO DAS EMISSÕES DE POLUENTES NA REGIÃO METROPOLITANA DE PORTO ALEGRE - RS
}

\author{
Ludmila Pochmann de Souza, Marcelo Felix Alonso, \\ Jonas da Costa Carvalho, Gustavo Copstein Cuchiara
}

\section{RESUMO}

Foi realizado o estudo do comportamento das concentrações de ozônio (O3) de superfície, monóxido de carbono $(\mathrm{CO})$, óxidos de nitrogênio (NOX) e hidrocarbonetos (HC) na Região Metropolitana de Porto Alegre (RMPA), através de simulações numéricas realizadas utilizando o PREP-CHEM (Preprocessor of trace gas and aerosol emission fields for regional and global atmospheric chemistry models) no modelo CCATT-BRAMS (Coupled Chemistry Aerosol and Tracer Transport model to the Brazilian developments on the Regional Atmospheric Modeling System). O domínio da modelagem foi implementado sobre uma área cobrindo a RMPA, dando ênfase às fontes antrópicas (móveis) e biogênicas. Para a validação dos resultados, foram realizadas comparações com dados de razão de mistura obtidos pelo monitoramento da qualidade do ar na RMPA.

\footnotetext{
ABSTRACT

Was conducted to study the behavior of the concentrations of surface ozone $(\mathrm{O} 3)$, carbon monoxide (CO), nitrogen oxides (NOX) and hydrocarbons (HC) in the Metropolitan Region of Porto Alegre (MRPA), through numerical simulations performed using the PREP-CHEM (PREProcessor of trace gas and aerosol emission fields for regional and global atmospheric CHEMistry models) in model CCATT BRAMS (Coupled Chemistry aerosol and Tracer Transport model to the Brazilian developments on the regional atmospheric Modeling System). The modeling domain was implemented on an area covering the MRPA, emphasizing the anthropogenic (mobile) and biogenic sources. To validate the results, they were compared with data obtained by the mixing ratio of air quality monitoring in the MRPA.
} 


\section{INTRODUÇÃO}

Um dos maiores problemas originados pela poluição do ar em áreas urbanas é o provocado por oxidantes fotoquímicos, sendo o principal deles o ozônio $\left(\mathrm{O}_{3}\right)$. O ozônio em superfície é formado por uma série de reações fotoquímicas complexas entre óxidos de nitrogênio $\left(\mathrm{NO}_{\mathrm{X}}\right.$ $\left.=\mathrm{NO}+\mathrm{NO}_{2}\right)$ e compostos orgânicos voláteis $(\mathrm{COV})$ na presença de luz solar (DERWENT et al., 2003). Os automóveis são as principais fontes de emissão dos precursores de $\mathrm{O}_{3}$ (ATKINSON-PALOMBO et al., 2006), não descartando a contribuição destes por fontes estacionárias (TAO et al., 2005) e biogênicas. Para o estudo de emissão, transporte, deposição e concentração de poluentes na atmosfera, a modelagem numérica é um dos métodos mais importantes para o estudo da poluição fotoquímica. Utilizando essa técnica, o presente estudo avaliou a influência de parâmetros meteorológicos na dispersão dos poluentes e verificou a variação diurna dos contaminantes primários e secundários das emissões da RMPA na formação e na destruição do ozônio troposférico em escala local e regional.

\section{MATERIAL E MÉTODO}

O domínio da modelagem foi implementado sobre uma área cobrindo toda a RMPA $\left(32^{\circ} \mathrm{S}\right.$ $\left.28.5^{\circ} \mathrm{S}\right)$ e $\left(53^{\circ} \mathrm{W}-49^{\circ} \mathrm{W}\right)$, no período de 05 a 13 de janeiro de 2009 . Para a preparação das emissões dos precursores do ozônio ( $\mathrm{CO}, \mathrm{NO}_{\mathrm{X}}$ e $\mathrm{HC}$ ), foi utilizada a ferramenta PREPCHEM (FREITAS et al., 2011). As simulações foram realizadas utilizando o modelo CCATT-BRAMS, e os resultados foram comparados com dados de razão de mistura medidos pela Fundação Estadual de Proteção Ambiental Henrique Luiz Roessler (FEPAM).

\section{RESULTADOS}

A formação da poluição fotoquímica ocorre com intensa radiação solar, ventos fracos, céu claro, alta temperatura, baixa umidade, camada limite estável (ARYA, 1999). De acordo com os dados meteorológicos do período (05 a 13 de janeiro de 2009), o tempo esteve 
caracterizado por duas condições distintas. Na primeira, entre os dias 05 e 09 de janeiro de 2009, a RMPA esteve sob a condição de céu claro e ventos fracos em superfície, favorecendo a formação e dispersão do $\mathrm{O}_{3}$ troposférico. Na segunda, entre os dias 09 e 13 de janeiro de 2009, as condições meteorológicas eram de céu encoberto e ventos moderados em superfície, o que dificulta tanto a formação quanto o transporte dos poluentes. A Fig. 1 apresenta alguns campos de direção e intensidade do vento simulados pelo modelo BRAMS. Observam-se ventos com intensidade fraca sobre o continente e com direção predominante de nordeste (NE), o que resulta no transporte da pluma formada sobre a RMPA em direção ao noroeste (NO) do RS.

(a)
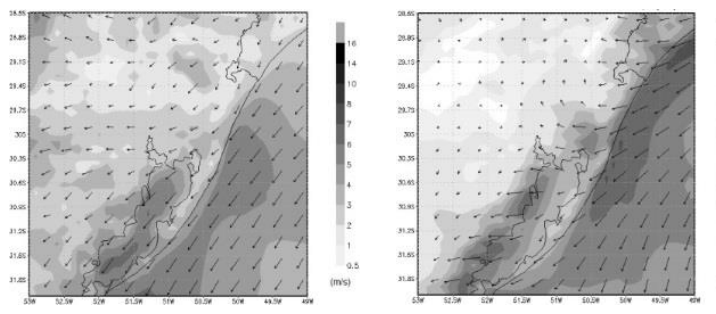

(b)

(c)

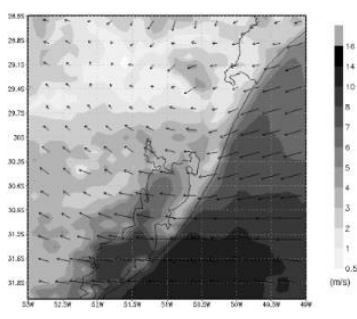

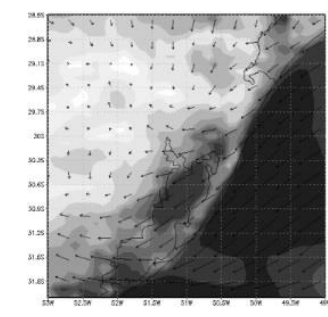

(d)

Figura 1 - Direção e velocidade do vento (a) 06/01/2009 as

00HL (b) 06/01/2009 as 15 HL (c) 10/01/2009 as 00HL (d) 10/01/2009 as 15HL.

Nas Fig. 2 a 4, apresentamos a comparação das simulações de razão de mistura com dados observados nas estações instaladas pela FEPAM em Porto Alegre e Esteio. As comparações são efetuadas entre os dias 5 e 13 de janeiro de 2009 para o $\mathrm{O}_{3}$ e seus precursores (CO, HC e $\mathrm{NO}_{\mathrm{X}}$. 

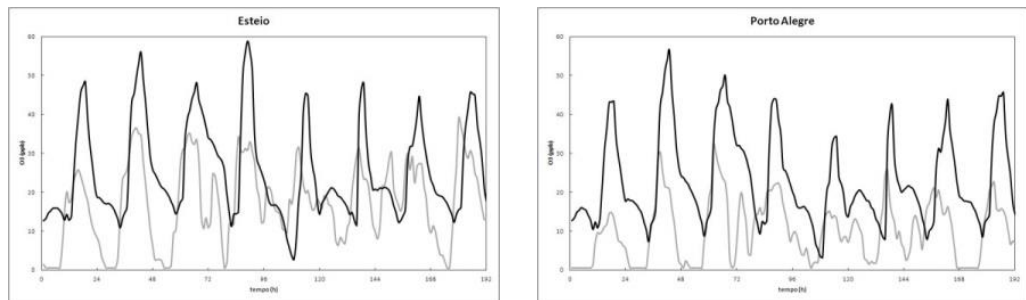

Figura 2 - Razão de mistura de $\mathrm{O}_{3}$ simulada (preto) e observada (cinza) nas cidades de Esteio (esquerda) e Porto Alegre (direita).
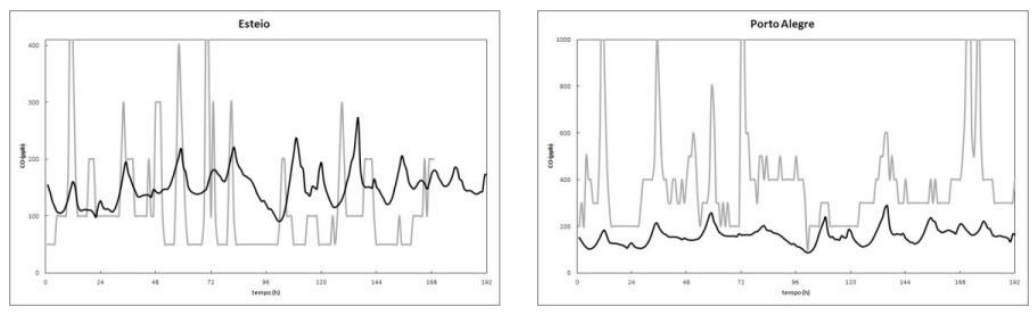

Figura 3 - Razão de mistura de CO simulada (preto) e observada (cinza) nas cidades de Esteio (esquerda) e Porto Alegre (direita).
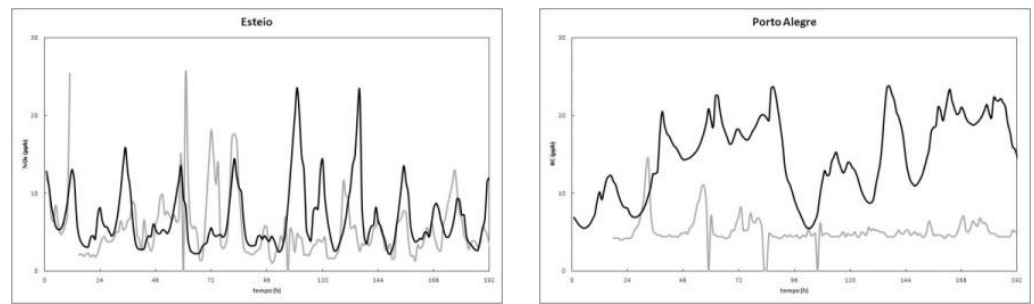

Figura 4 - Razão de mistura simulada (preto) e observada (cinza) de NOx em Esteio (esquerda) e HC em Porto Alegre (direita).

\section{CONCLUSÕES}

Os resultados das simulações mostram que modelo CCATT-BRAMS simula satisfatoriamente o padrão de ciclo diurno de $\mathrm{O}_{3}$ e de seus precursores $(\mathrm{CO}, \mathrm{HC}$ e $\mathrm{NO}$ ). Nota-se que o modelo superestima os valores observados de $\mathrm{O}_{3}$ e $\mathrm{HC}$ e subestima os valores observados de CO para a estação de Porto Alegre. Em geral, os problemas encontrados nas comparações dos resultados do modelo, estão nas incertezas com relação às estimativas das emissões a partir das fontes móveis e na especiação dos hidrocarbonetos não-metanos. Tais fatos acabam limitando o entendimento da relação entre emissões e razão de mistura de poluentes. 


\section{AGRADECIMENTOS}

Ao Grupo de Modelagem da Atmosfera e Interfaces (GMAI/INPE). A Coordenação de Aperfeiçoamento de Pessoal de Nível Superior (CAPES) pelo financiamento e à FEPAM pelos dados de qualidade do ar.

\section{BIBLIOGRAFIA}

ATKINSON-PALOMBO, C.; MILLER,J.A.; BALLING JR.; ROBERT, C. QUANTIFYING the ozone " weekend effect " at various locations in Phoenix, Arizona. Atmospheric Environment., n 40, p. 7644-7658, 2006.

ARYA, P. S. Air Pollution, Meteorology and Dispersion, Oxford University Press, New York, 1999.

DERWENT, R.G.; JENKIN, M.E.; SAUNDERS, S.M.; PILLING, M.J.; SIMMONDS, P.G.; PASSANT, N.R.; DOLLARD, G.J.; DUMITREAN,P.; KENT, A.. Photochemical ozone formation in north west Europe and its control. Atmospheric Environment., 37, 1983. 2003. FREITAS, S. R.; LONGO, K. M.; ALONSO, M. F.; PIRRE, M., MARÉCAL, V., GRELL, G.; STOCKLER, R.; DA FONSECA, R.M.; SÁNCHEZ GÁCITA, M.. A pre-processor of trace gases and aerosols emission fields for regional and global atmospheric chemistry models, Geosci. Model Dev., n 4, p. 419-433, doi:10.5194/gmd-4-419- 2011, 2011.

TAO, Z.; LARSON, S. M.; WILlIAMS, A.; CAUGHEY, M.; WUEBBLES, D. J.. Area, mobile, and point source contributions to ground level ozone : a summer simulation across the continental USA. Atmospheric Environment, 39, 1869-1877, 2005. 